\title{
Evaluation of Push out Bond Strength of Different Endodontic Sealers with Different Obturation Techniques
}

\section{Yousra Aly and Sherif El Shershaby}

Restorative and Dental Materials Department, National Research Centre, 33 El-Buhouth St., 12622 Dokki, Giza, Egypt.

Received: 20 June 2020 / Accepted 30 July 2020 / Publication date: 20 August 2020

\begin{abstract}
Aim: This study aimed to compare the push-out bond strength of two different endodontic sealers with different obturation techniques to root dentin. Methods: Thirty single rooted extracted teeth were selected and instrumented using ProTaper rotary files. The canals were assigned into three equal groups based upon the sealer and technique used. Group I: Epoxy resin-based sealer (AH Plus Sealer) with single cone gutta percha, Group II: Epoxy resin-based sealer (AH Plus Sealer) with continuous wave compaction (CWC) technique and Group III: Bioceramic sealer (CeraSeal) with single cone bioceramic coated Bio GP Points. Roots were sectioned transversally at the thickness of $2 \mathrm{~mm}$ to obtain 3 sections (coronal, middle and apical $n=30$ per group). The specimens were subjected to pushout test using a Universal Test Machine at a loading speed of $0.5 \mathrm{~mm} / \mathrm{min}$. Results: The push-out bond strength of CeraSeal sealer when used with Bio GP Points was significantly higher than that of AH Plus sealer in single cone technique; While the bond strength of CeraSeal sealer was higher but not statistically significantly with AH Plus sealer in continuous wave compaction (CWC) technique. Conclusions: The bioceramic sealer Ceraseal combined with Bio GP Points showed a significantly higher bond strength than single cone AH Plus in coronal, middle and apical thirds.
\end{abstract}

Keywords: push out test, single cone, bioceramic sealer, AH Plus

\section{Introduction}

The main targets of endodontic therapy are proper cleaning and shaping and three dimensional obturation of the root-canal system. The most widely used and accepted obturation material is the gutta percha. It is well known by its biocompatibility, inertness, dimensional stability and ease of removal for post placement or retreatment (Schilder, 1967).

Many studies demonstrated that there is a direct relationship between the quality of root canal obturation and treatment success (Tavares et al., 2009). Failure of achievement of strong bond between root canal walls and filling materials may lead to bacterial infusion towards the apical third of the root causing apical periodontitis (Gillen et al., 2011).

The characteristics of the material as well as the type of obturation technique used may affect the obturation quality. In this study, two different obturation technique were used: The thermoplastic technique and the single cone technique (SC). Obturation of oval or irregular root canals is very challenging. For these types of root canals, many studies recommended the use of the thermoplastic obturation techniques due to its many advantages (Celikten et al., 2016). The single-cone (SC) technique became widely used due to its low cost, ease of handling and short procedure time (Tasdemir et al., 2009 ; Cavenago et al., 2012).

New bioceramic coated gutta percha was introduced into the market by SureEndo Corporation, korea. The manufacturers claim that it can create monoblock obturation in root canal when used with bioceramic sealer (Bio GP Points Pamphlet, 2019).

In this study, CeraSeal (Meta Biomed Co., Ltd. Korea, Republic) which is a new bioceramic sealer was used. It is in the form of a flowable paste that can be immediately applied inside the root canal. The manufacturers claim that it has a unique stability; never shrink or expand. Moreover, they claim that it has excellent sealing ability so, the single cone technique obturation can be performed. (CeraSeal Pamphlet, 2019). 
The push-out bond strength is a valuable test to predict the prognosis of the bond of the root canal sealer and core material to dentin (Pane et al., 2013).

In the present study, we evaluated and compared the Push out bond strength to root dentin of recently introduced bioceramic sealer (CeraSeal) combined with bioceramic coated gutta percha (Bio GP points), and AH Plus sealer (epoxy resin-based sealer) used with single cone technique or continuous wave compaction (CWC).

\section{Materials and Methods}

\section{Specimen selection:}

Thirty single rooted freshly extracted human teeth with mature apices, without any root caries, root fracture or resorption were selected. Teeth were carefully cleaned with curettes to remove any soft tissue remnants, placed in $2.5 \%$ sodium hypochlorite NaOCL for one hour to allow for surface disinfection and then stored in saline solution until instrumentation.

\section{Specimen preparation:}

The teeth were decoronated to create root sample with standardized length of $16 \mathrm{~mm}$ using a safe sided diamond disk mounted in a low speed handpiece under water coolant. The working length was measured by subtracting $1 \mathrm{~mm}$ from the length recorded when the tip of a \# $15 \mathrm{~K}$-file was visible at the apical foramina. The root canals were prepared using Protaper rotary system to an apical preparation size F3. Irrigation of the canals with $1 \mathrm{ml}$ of $2.5 \% \mathrm{NaOCL}$ was done between each instrument. After completion of the instrumentation, the smear layer was removed with $3 \mathrm{ml}$ of $17 \%$ EDTA for 3 minutes followed by $3 \mathrm{ml} 5.25 \% \mathrm{NaOCl}$. Finally, the canal was flushed with $3 \mathrm{ml}$ distilled water then dried with paper points. The teeth were then divided into three groups.

Group I: Epoxy resin based (AH Plus sealer) with single cone gutta percha (F3). An appropriate amount of base and catalyst was squeezed onto a mixing plate. They were mixed with the spatula for 15-20 seconds or until creamy and homogeneous consistency was reached. Gutta percha points Protaper F3 were coated with the AH Plus sealer and placed up to the working length. The cone was then seared off at the orifice level.

Group II: Protaper F3 gutta-percha cone was placed 1mm short of the WL and examined for tug back. The sealer was applied to the canal walls using a $\# 40 \mathrm{k}$-file. The tip of the master gutta percha cone was coated with sealer and placed in the canal. A heat source (Fast pack, Eighteeth, china) at $180^{\circ} \mathrm{C}$ was used. A medium- size tip was placed on the fast pack heat source with a rubber stop set at $5 \mathrm{~mm}$ short of the WL. The tip was allowed to cool for 8 seconds, and a single burst of heat was applied for 1 second and the tip was removed. After removing the coronal and middle portions of the fillings, the softened gutta-percha was compacted using the widest plugger, which led to perfect obturation of the apical area and accessory canals. A cordless gutta percha obturation gun (Fast Fill, Eightheeth, China) with \#23-gauge needle was set on $200^{\circ} \mathrm{C}$ and gutta-percha pellets (Meta Biomed Co. Ltd, Cheongju City, Chungbuk, Korea) was inserted into the root canal up to the level of the already placed gutta-percha in the apical portion. Backfill was completed up to the root canal orifice. During backfill, Gun needle was pushed back simultaneously by gutta-percha being filled. The heated gutta-percha was compacted using a bigger plugger, which led to complete obturation of the root canal system.

Group III: (CeraSeal) was placed into the canal with the provided syringe tip. The syringe bar was pushed slowly into the canal to fill it, and then pulled out slowly out of the canal. The bioceramic coated gutta-percha point (F3) was then inserted into the root canal. The upper part of gutta percha was then cut and removed by heated plugger. The excess sealer was removed by water-soaked cotton pellets.

The samples were coronally restored with Cavit G (3M ESPE, Germany) and stored in the incubator at $95 \%$ relative humidity and $37^{\circ} \mathrm{C}$ for one week. 


\section{Push out bond strength test}

Teeth were embedded in chemical cured acrylic resin and then cross- sectioned using IsoMet 4000 microsaw Buehler USA mounting diamond disk $0.6 \mathrm{~mm}$ thickness at speed $2500 \mathrm{rpm}$ and feeding rate $10 \mathrm{~mm} / \mathrm{min}$ under water cooling. $2 \mathrm{~mm}$ thick slices of apical-root portion for assessment of push out bond strength. Apical and coronal aspects of each sample were photographed and examined using stereomicroscope (Nikon MA100 Japan) confirm absence of dentin cracks or voids of the filling materials. The filling material was then loaded with a $0.9 \mathrm{~mm}$ diameter stainless steel plunger selected. The plunger was mounted on the upper part of a universal testing machine (Instron universal testing machine model 3345 England data recorded using computer software Bluehill 3 version 3.3). The samples were aligned over a support jig in an apical to coronal direction to avoid any constriction interference. The tests were conducted at a cross head speed of 0.5 min- 1 using a $500 \mathrm{~N}$ load cell. The highest value recorded was taken as the push-out bond strength.

The area under load was calculated by:

Area $=$ circumference of restoration $\times$ thickness.

The push-out value in MPa was calculated from force $(\mathrm{N})$ divided by area in $\mathrm{mm}^{2}$.

\section{Statistical analysis}

The mean and standard deviation values were calculated for each group in each test. Data were explored for normality using Kolmogorov-Smirnov and Shapiro-Wilk tests, data showed parametric (normal) distribution.

Repeated measure ANOVA was used to compare between more than two groups in related samples. Paired sample t-test was used to compare between two groups in related samples. One-way ANOVA followed by post hoc Tukey test was used to compare between more than two groups in nonrelated samples.

Two-way ANOVA test was used to test the interactions between different variables.

The significance level was set at $\mathrm{P} \leq 0.05$. Statistical analysis was performed with $\mathrm{IBM}^{\circledR}$ SPSS ${ }^{\circledR}$ Statistics Version 20 for Windows

\section{Results}

Regarding the effect of Thirds: (Table 1 and figure 1).

In Group I (AH Plus sealer with single cone Protaper F3), no statistically significant difference was found between (Coronal), (Middle) and (Apical) groups where $(p=0.333)$. Coronal third recorded the highest mean value followed by (Apical) third, then (Middle) third.

In Group II (Ceraseal with CWC technique), a statistically significant difference was found between (Coronal), (Middle) and (Apical) groups where ( $p=0.005)$.

A statistically significant difference was found between (Coronal) and (Apical) groups where $(p=0.004)$. Coronal third recorded the highest mean value followed by (Middle) third, then (Apical) third.

In Group III (Ceraseal with single cone F3 Bioceramic gutta percha), A statistically significant difference was found between (Coronal) and (Apical) groups where $(p=0.013)$. The highest mean value was found in (Coronal) third followed by (Middle) third, then (Apical) third.

Regarding the effect of groups: (Table 1 and figure 2).

\section{a) Coronal:}

There was a statistically significant difference between (Group I), (Group II) and (Group III) where $(p<0.001)$.

A statistically significant difference was found between (Group I) and each of (Group II) and (Group III) where $(p<0.001)$.

(Group III) recorded the highest mean value, followed by (Group II) then (Group I). 


\section{b) Middle:}

There was a statistically significant difference between (Group I), (Group II) and (Group III) where $(p<0.001)$.

A statistically significant difference was found between (Group I) and each of (Group II) and (Group III) where $(p=0.001)$.

(Group III) recorded the highest mean value followed by (Group II), then (Group I).

\section{c) Apical:}

There was a statistically significant difference between (Group I), (Group II) and (Group III) where $(p<0.001)$.

A statistically significant difference was found between (Group I) and each of (Group II) and (Group III) where $(p=0.016)$ and $(\mathrm{p}<0.001)$ respectively.

(Group III) recorded the highest mean value followed by (Group II), then (Group I).

Table 1: The mean, standard deviation (SD) values of push out bond strength of different groups in different thirds.

\begin{tabular}{|c|c|c|c|c|c|c|c|}
\hline \multirow{3}{*}{ Variables } & \multicolumn{7}{|c|}{ Push out bond strength } \\
\hline & \multicolumn{2}{|c|}{ Group I } & \multicolumn{2}{|c|}{ Group II } & \multicolumn{2}{|c|}{ Group III } & \multirow[t]{2}{*}{ p-value } \\
\hline & Mean & SD & Mean & SD & Mean & SD & \\
\hline Coronal & $11.00^{\mathrm{aB}}$ & 2.52 & $20.12^{\mathrm{aA}}$ & 2.04 & $21.14^{\mathrm{aA}}$ & 1.57 & $<0.001 *$ \\
\hline Middle & $9.00^{\mathrm{aB}}$ & 1.26 & $16.14^{\mathrm{abA}}$ & 2.17 & $16.23^{\mathrm{abA}}$ & 2.92 & $<0.001 *$ \\
\hline Apical & $9.21^{\mathrm{aB}}$ & 1.74 & $13.07^{\mathrm{bA}}$ & 1.46 & $15.72^{\mathrm{bA}}$ & 2.23 & $<0.001 *$ \\
\hline p-value & \multicolumn{2}{|c|}{$0.333 n s$} & \multicolumn{2}{|c|}{$0.005 *$} & \multicolumn{2}{|c|}{ 0.024* } & \\
\hline
\end{tabular}

Means with different small letters in the same column indicates significant difference, means with different capital letters in the same row indicates significant difference *; significant $(\mathrm{p}<0.05) \quad$ ns; non-significant $(\mathrm{p}>0.05)$.

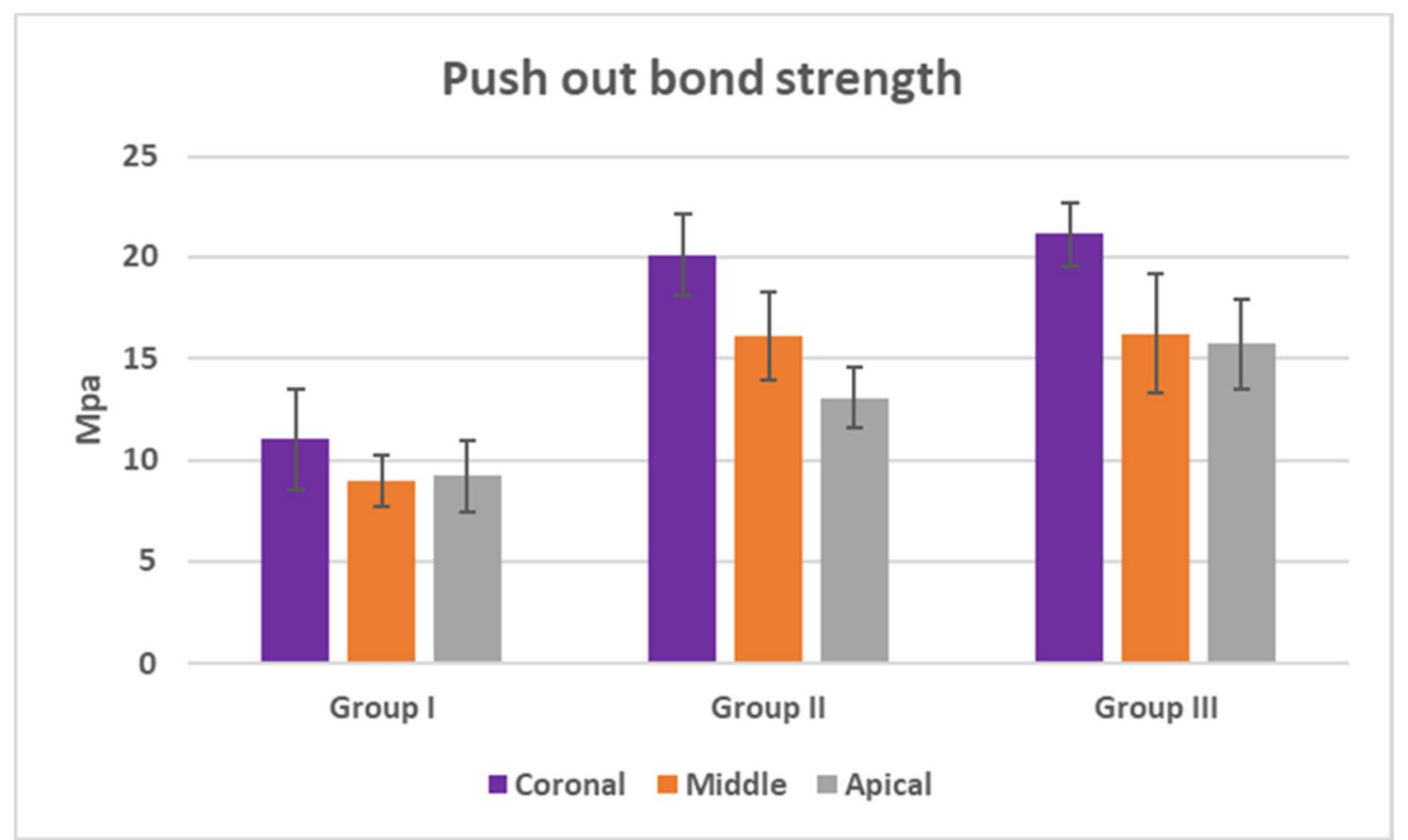

Fig. 1: Bar chart representing push out bond strength of different groups 


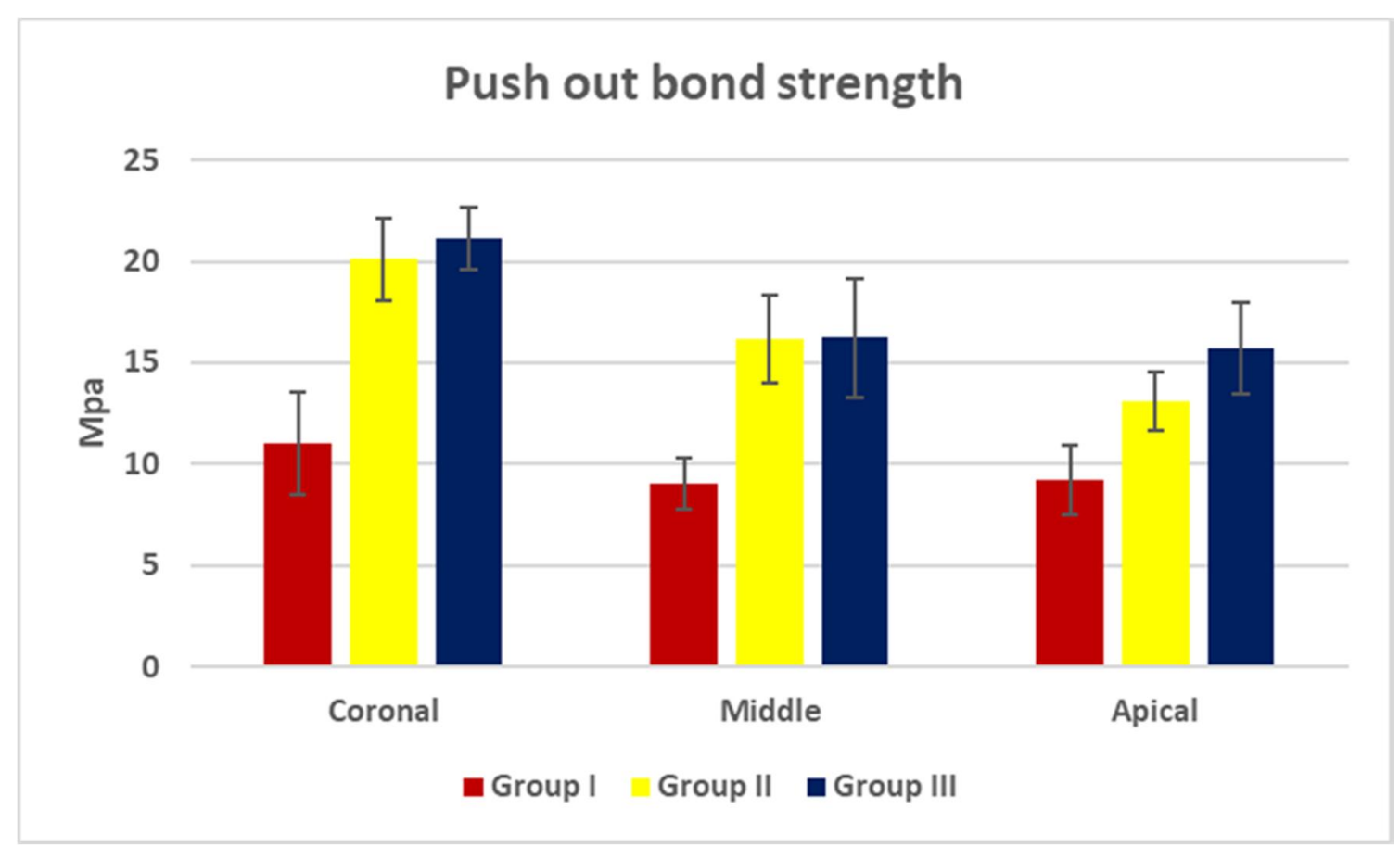

Fig. 2: Bar chart representing push out bond strength of different third

\section{Discussion}

The essential factors for successful endodontic treatment are proper cleaning and shaping of the root canals and the creation by the filling materials of a single block configuration in the canal space. Because of the poor adhesiveness of gutta-percha to root canal walls, the use of sealers has been considered mandatory. The major function of root canal sealers is to form a bond between the root filling material and the canal walls.

That's why, there is a continuous improvement in root canal filling materials and bioceramic materials are now becoming widely used. The Ceraseal sealer (Meta Biomed Co., Ltd. Korea, Republic) is one of the bioceramic sealants that are recently introduced into the market.

In this study, obturation was done by sealers and core materials in all the samples. Two different obturation technique were used: The continuous wave compaction technique (CWC) was used because it showed a better filling ability of canal irregularities as well as lateral canals (Schafer et al., 2016); and the single cone technique (SC) as SC technique is known to be simple and fast, although in some studies it showed inadequate obturation in oval root canals (Robberecht et al., 2012; and Horsted et al., 2007).

In SC technique, in order to achieve a strong bond between filling materials and root canal, a tapered gutta-percha cone matching the prepared canal shape is used. Nowadays, many instrumentation systems are based on this technique.

The bond strength between sealer and canal walls is commonly evaluated by the push-out test. It is based on the application of tensile load vertically to the long axis of the root till dislodgement of the filling material (Collares et al., 2016).

Although, bond strength testing may not be able to completely test the sealers as in the clinical condition, but it is a way to compare between different sealers or obturation techniques (Madhuri et al., 2016)

In the present study, Ceraseal BC Sealer showed the highest bond strength with a statistically significant difference $(\mathrm{P}=0.0001)$. This may be due to the formation of chemical bond with dentine through hydroxyapatite production during setting. This clarify the cause of the true self-adhesive nature of this bioceramic sealer. Moreover, it diffuses easily into the dentinal tubules which results in good adaptation and hermetic seal (Zhang et al., 2009). 
In the presence of moisture and the contact with phosphate from tissue fluids, the release of calcium hydroxide from di- and tricalcium silicate cements leads to a precipitation of calcium phosphate or calcium carbonate on the material's surface (Sarkar et al.,2005).

Moreover, partial reaction with phosphate leads to hydroxyapatite formation on a calcium silicate sealer's surface (Zhang et al., 2009).

This may explain the bioactive ability of bioceramic sealers (Torabinejad, 2014). Furthermore, an interfacial layer named "mineral infiltration zone" at the dentin wall is formed by calcium silicates. The strong alkaline effects of the calcium silicate cement's leads to denaturation of the collagen fibers of the interfacial dentin (Atmeh et al., 2012).

Thus, a porous structure that facilitates the permeation of high concentrations of $\mathrm{Ca}_{2}+, \mathrm{OH}-$, and $\mathrm{CO}_{3} 2-$ ions is formed, resulting in increased mineralization in this region (Atmeh et al., 2012; Watson et al., 2014).

The adhesion obtained between calcium silicate-based materials and dentin is mainly due to this chemical interaction at the interfacial dentin along with a mechanical interlocking by tag-like structures (Atmeh et al., 2012; Kaup et al., 2015).

Also, the Bioceramic coated gutta percha cones has the capability to absorb water from the tooth environment and expand in the lateral direction only to hermetically seal the root canal. Delong et al. (2015) in his study concluded that Bioceramic sealer showed highest bond strength than AH plus sealer when used in a single cone technique.

In our study AH Plus sealer showed the lowest bond strength. This was in disagreement with Gurgel-Filho and Martins (2014) who concluded that push-out bond strength of AH Plus combined with gutta-percha were higher than Endofill sealer and MTA Fill apex core combination this may be due different in methodology.

Group II (AH Plus sealer with CWC technique) showed non-significant lower bond strength than group III in which obturation was done with CeraSeal and single cone bioceramic coated gutta percha. Meanwhile, Group II showed a statistically significant higher bond strength than Group I. This may be due to the obturation technique used in this group.

\section{Conclusion}

The bioceramic sealer (Ceraseal) combined with single cone coated bioceramic gutta percha Bio GP Points had a better bond strength than that of AH Plus when used with single cone technique or with Continuous wave compaction technique CWC.

\section{References}

Atmeh, A.R., E.Z. Chong, G. Richard, F. Festy and T.F. Watson, 2012. Dentin-cement interfacial interaction: calcium silicates and polyalkenoates. J. Dent. Res., 91:454-9.

Bio GP Points Pamphlet, 2019. Sure Dent Corporation, Korea. www.suredent.com

Cavenago, B.C., M.A. Duarte, R. Ordinola-Zapata, et al., 2012. Interfacial adaptation of an epoxyresin sealer and a self-etch sealer to root canal dentin using the System B or the single cone technique. Braz. Dent. J., 23:205-11.

Celikten, B., C.F. Uzuntas, A.I. Orhan, et al., 2016. Evaluation of root canal sealer filling quality using a single-cone technique in oval shaped canals: an in vitro Micro-CT study. Scanning, 38:133-40.

CeraSeal Pamphlet, 2019. Meta Biomed Co., Ltd. Korea, Republic.

Collares, F.M., F.F. Portella, S.B. Rodrigues, R.K. Celeste, V.C.B. Leitune, and S.M.W. Samuel, 2016. The influence of methodological variables on the push-out resistance to dislodgement of root filling materials: a meta-regression analysis. Int. Endod. J, 49(9):836-49.

DeLong, C., J. He, and K.F. Woodmansey, 2015. The effect of obturation technique on the push-out bond strength of calcium silicate sealers. J. Endod., 41:385-8.

Gillen, B.M., S.W. Looney, L.S. Gu, et al., 2011. Impact of the quality of coronal restoration versus the quality of root canal fillings on success of root canal treatment: a systematic review and meta-analysis. J. Endod., 37:895-902.

Gurgel-Filho, E.D. and F. Martins, 2014. Comparative evaluation of push-out bond strength of an MTA based root canal sealer. Braz. J. Oral Sci., 13:114-7. 
Horsted-Bindslev, P., M.A. Andersen, M.F. Jensen, et al., 2007. Quality of molar root canal fillings performed with the lateral compaction and the single-cone technique. J. Endod., 33:468-71.

Kaup, M., C. Dammann, E. Schäfer, and T. Dammaschke, 2015. Shear bond strength of biodentine, ProRoot MTA, glass ionomer cement and composite resin on human dentine ex vivo. Head Face Med., 19:11-4.

Madhuri, G., S. Varri, N. Bolla, P. Mandava, L. Akkala and J. Shaik, 2016. Comparison of bond strength of different endodontic sealers to root dentin: An in vitro push-out test. J of Conservative Dentistry, 19; 461-464.

Pane, E.S., J.E. Palamara, and H.H. Messer, 2013.Critical evaluation of the push-out test for root canal filling materials. J. Endod., 39:669-73.

Robberecht, L., T. Colard, and A. Claisse-Crinquette, 2012. Qualitative evaluation of two endodontic obturation techniques: tapered single-cone method versus warm vertical condensation and injection system an in vitro study. J. Oral Sci., 54:99-104

Sarkar, N.K., R. Caicedo, P. Ritwik, R. Moiseyeva, and I. Kawashima, 2005. Physicochemical basis of the biologic properties of mineral trioxide aggregate. J. Endod; 31:97-100.

Schafer, E., C. Schrenker, J. Zupanc, and S. Burklein, 2016. Percentage of gutta-percha filled areas in canals obturated with cross-linked gutta-percha core-carrier systems, single-cone and lateral compaction technique. J. Endod., 42:294-8.

Schilder, H., 1967. Filling root canals in three dimensions. Dent Clin North Am; 11:723-44.

Tasdemir, T., C. Yesilyurt, K.T. Ceyhanli, et al., 2009. Evaluation of apical filling after root canal filling by 2 different techniques. J. Can. Dent. Assoc., 75:201a-201d.

Tavares, P.B., E. Bonte, T. Boukpesso, et al., 2009. Prevalence of apical periodontitis in root canaltreated teeth from an urban French population: influence of the quality of root canal fillings and coronal restorations. J Endod., 35:810-3.

Torabinejad, M., 2014. Calcium silicate-based cements. In: Torabinejad M, editor. Mineral trioxide aggregate: properties and clinical applications. Ames: Wiley Blackwell, 281-332.

Watson, T.F., A.R. Atmeh, S. Sajini, R.J. Cook, and F. Festy, 2014. Present and future of glassionomers and calcium-silicate cements as bioactive materials in dentistry: biophotonicsbased interfacial analyses in health and disease. Dent. Mater., 30:50-61.

Zhang H., Y. Shen, N. D. Ruse, and M. Haapasalo, 2009. Antibacterial activity of endodontic sealers by modified direct contact test against Enterococcus faecalis. Journal of Endodontics, 35:(7) 1051-1055.

Zhang W., Z. Li and B. Peng, 2009. Assessment of a new root canal sealer's apical sealing ability," Oral Surgery, Oral Medicine, Oral Pathology, Oral Radiology and Endodontics, 107: (6) e79-e82. 